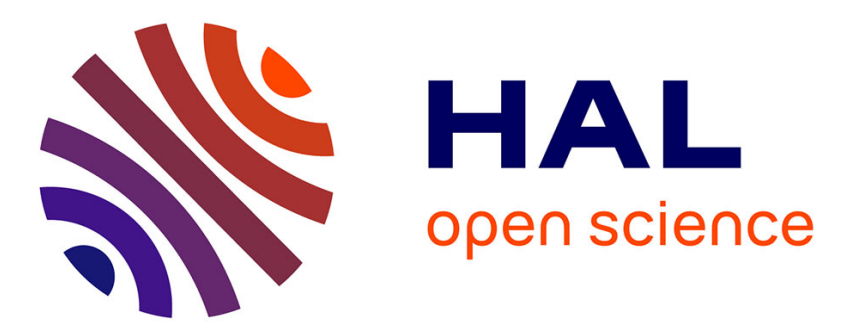

\title{
Observer Design for Time Delay Nonlinear Triangular Systems
}

\author{
Boubekeur Targui, Carlos Manuel Astorga-Zaragoza, Miloud Frikel, \\ Mohammed M'Saad, Eric Magarotto
}

\section{- To cite this version:}

Boubekeur Targui, Carlos Manuel Astorga-Zaragoza, Miloud Frikel, Mohammed M'Saad, Eric Magarotto. Observer Design for Time Delay Nonlinear Triangular Systems. 18th IFAC World Congress, Aug 2011, Milan, Italy. 6 p. hal-01063155

\section{HAL Id: hal-01063155 \\ https://hal.science/hal-01063155}

Submitted on 11 Sep 2014

HAL is a multi-disciplinary open access archive for the deposit and dissemination of scientific research documents, whether they are published or not. The documents may come from teaching and research institutions in France or abroad, or from public or private research centers.
L'archive ouverte pluridisciplinaire $\mathbf{H A L}$, est destinée au dépôt et à la diffusion de documents scientifiques de niveau recherche, publiés ou non, émanant des établissements d'enseignement et de recherche français ou étrangers, des laboratoires publics ou privés. 


\title{
Observer design for time delay nonlinear triangular systems
}

\author{
B. Targui*, CM. Astorga-Zaragoza**, M. Frikel*, \\ M. M'Saad*, E. Magarotto* \\ * Groupe de Recherche en Informatique, Image, Automatique et \\ Instrumentation de Caen, UMR 6072, 6 Boulevard du Marechal Juin, \\ 14050, Caen Cedex, France. (Email: \\ boubekeur.targui@greyc.ensicaen.fr, Tel.+33(0)231452712, Fax: \\ +33(0)2 31452698 ) \\ ** Centro Nacional de Investigacin y Desarrollo Tecnológico, Interior \\ Internado Palmira s/n, Col. Palmira, C.P. 62490, Cuernavaca, Mor., \\ Mexico
}

\begin{abstract}
In this work an observer for a class of delayed nonlinear triangular systems with multiple and simple time-delay is proposed. The main feature of this observer is that it is easy to implement because of its constant gain. First, the observer synthesis is presented for nonlinear systems with variable time-delay and is extended for nonlinear systems with multiple time-delay. The observer performance is evaluated through numerical simulations.
\end{abstract}

Keywords: nonlinear time-delay systems, nonlinear observers, triangular forms.

\section{INTRODUCTION}

In the recent years time-delayed systems have been investigated extensively because of the delay phenomenon is often encountered in various engineering systems such as mechanical and electrical systems, communication networks, among others. The source of a time-delay may be due to the nature of the system or induced into the system due to the transmission delays associated to other components interacting with the system. A time-delay may be the origin of instability or oscillations in a system. For this reason, many researchers are devoted to investigate the different fields of automatic control for time-delayed systems, such as stability, observability, controllability and system identification, among others. For instance, in Richard (2003), different control approaches for delayed systems are presented.

The case of observer design for state estimation of nonlinear systems is treated by many authors from a theoretical and practical point of view, see for instance, Gauthier et al. (1992), Gauthier and Kupka (1994), Targui et al.(2002), Farza et al. (2004). The observer synthesis for triangular nonlinear systems is related to the notion of uniform observability, for instance, the authors in Targui et al.(2002), propose a high-gain observer with constant gain for nonlinear systems having a triangular structure and a single output. In Targui et al. (2001), a generalization for multipleoutput systems is presented. A lot of researchers, such as Darouach (2001), Hou (2002), Wang et al. (2002), have extensively investigated the case of observer synthesis for delayed systems. For instance, in Germani et al. (2002), a chain of observation algorithms reconstructing the system state based on delayed measurements of the process output is proposed. In Ibrir (2009), an adaptive observer for time- delay nonlinear systems in triangular form is presented. This paper is organized as follows. Section 2 is devoted to preliminaries and the problem formulation. Section 3 describes the observer synthesis for a class of nonlinear systems with a signal variable time-delay. An extension of this observer for nonlinear systems with multiple and variable delays is presented in Section 4. In Section 5 numerical simulations are presented in order to evaluate the observer performance and finally conclusions are discussed in Section 6.

\section{PRELIMINARIES AND PROBLEM FORMULATION}

Consider the following non-linear delayed system:

$$
\left\{\begin{array}{l}
\dot{x}_{1}=f_{1}\left(u, u_{\tau}, x_{1}, x_{1 \tau}, x_{2}\right) \\
\dot{x}_{2}=f_{2}\left(u, u_{\tau}, x_{1}, x_{1 \tau}, x_{2}, x_{2 \tau}, x_{3}\right) \\
\vdots \\
\dot{x}_{i}=f_{i}\left(u, u_{\tau}, x_{1}, x_{1 \tau}, x_{2}, x_{2 \tau}, \ldots, x_{i}, x_{i \tau}, x_{i+1}\right) \\
\vdots \\
\dot{x}_{n-1}=f_{n-1}\left(u, u_{\tau}, x_{1}, x_{1 \tau}, \ldots, x_{n-1}, x_{n-1 \tau}, x_{n}\right) \\
\dot{x}_{n}=f_{n}\left(u, u_{\tau}, x, x_{\tau}\right) \\
y=x_{1}
\end{array}\right.
$$

where $u(t) \in \mathbb{R}^{m}$ and $y \in \mathbb{R}$ are the input and the output of the system. $x(t)=\left[\begin{array}{c}x_{1}(t) \\ x_{2}(t) \\ \vdots \\ x_{n}(t)\end{array}\right] \in \mathbb{R}^{n}$ is the state vector.

Henceforth the next notations are used 


$$
\begin{gathered}
x_{\tau}(t)=x(t-\tau(t))=\left[\begin{array}{c}
x_{1}(t-\tau(t)) \\
x_{2}(t-\tau(t)) \\
\vdots \\
x_{n}(t-\tau(t))
\end{array}\right]=\left[\begin{array}{c}
x_{1 \tau} \\
x_{2 \tau} \\
\vdots \\
x_{n \tau}
\end{array}\right] \in \mathbb{R}^{n}, \\
u_{\tau}(t)=u(t-\tau(t)),
\end{gathered}
$$

where $\tau(t)$ a known variable delay and $x(t)=\phi(t)$ for $t \leq \tau$. $\phi(t)$ is the initial condition belonging to a Banach space and $\|\cdot\|$ represents the Euclidian norm of a vector. System (1) can be written in the following form:

$$
\left\{\begin{array}{l}
\dot{x}(t)=f\left(u(t), u_{\tau}(t), x(t), x_{\tau}(t)\right) \\
y(t)=C x(t)
\end{array}\right.
$$

where

$$
f=\left[\begin{array}{c}
f_{1}\left(u, u_{\tau}, x_{1}, x_{1 \tau}, x_{2}\right) \\
f_{2}\left(u, u_{\tau}, x_{1}, x_{1 \tau}, x_{2}, x_{2 \tau}, x_{3}\right) \\
\vdots \\
f_{n-1}\left(u, u_{\tau}, x_{1}, x_{1 \tau}, \ldots, x_{n-1}, x_{n-1 \tau}, x_{n}\right) \\
f_{n}\left(u, u_{\tau}, x, x_{\tau}\right)
\end{array}\right]
$$

and $C$ is a $(1 \times n)$ matrix:

$$
C=[1,0, \ldots, 0]
$$

The following assumptions are introduced:

Assumption 1 (A1). The functions $f_{i} ; i=1, \ldots, n$ are globally Lipschitz w.r.t. $x$ and $x_{\tau}$, then $\exists c>0$ and $\forall x(t)$, $y(t), x(t-\tau), y(t-\tau) \in \mathbb{R}^{n}$ such that:

$\left\|f\left(u, u_{\tau}, x, x_{\tau}\right)-f\left(u, u_{\tau}, y, y_{\tau}\right)\right\| \leq c\left(\|x-y\|+\left\|x_{\tau}-y_{\tau}\right\|\right)$

Assumption 2 (A2). Assume that the functions $f_{i}(),. i=$ $1, \ldots, n-1$ satisfy.

$$
\begin{aligned}
0<\alpha<a_{i}= & \frac{\partial f_{i}}{\partial x_{i+1}}(u, \xi) \leq \beta \\
& i=1, \ldots, n-1, \forall \xi \in R^{n}
\end{aligned}
$$

Assumption 3 (A3). The variable delay $\tau(t)$ is bounded, e.g. $0<\tau(t) \leq \tau_{\max }$, it is differentiable and satisfies $\dot{\tau}(t) \leq \mu<1$.

The following notations are employed:

- $A$ is a $(n \times n)$ matrix having the following form:

$$
A=\left(\begin{array}{ccccc}
0 & a_{1} & 0 & \ldots & 0 \\
0 & 0 & a_{2} & & \vdots \\
\vdots & & \ddots & 0 \\
0 & \ldots & 0 & a_{n-1} \\
0 & \ldots & 0 & 0
\end{array}\right)
$$

where the functions $a_{i} ; i=1, \ldots, n-1$ are defined in Equation (4) and each one satisfies Assumption (A2).

- $S$ is a $(n \times n)$ constant symmetric positive-definite matrix having the form:

$$
S=\left(\begin{array}{ccccc}
s_{11} & s_{1} & 0 & & 0 \\
s_{1} & s_{22} & \ddots & & 0 \\
0 & \ddots & & & \vdots \\
\vdots & & & \ddots & s_{n-1} \\
0 & \ldots & 0 & s_{n-1} & s_{n n}
\end{array}\right)
$$

where $s_{i i}>0$ and $s_{j}<0 ; i=1, \ldots, n ; j=1, \ldots, n-1$.

- Define the $(n \times n)$ diagonal matrix $\Lambda_{\rho}$ as

$$
\Lambda_{\rho}=\operatorname{diag}\left[\rho, \rho^{2}, \ldots, \rho^{n}\right]
$$

where $\rho$ is a strictly positive real number.

Lemma. (Targui et al.(2002)). There exist an $n \times n$ constant symmetric positive definite matrix $S$ having the form given in Equation (6) and a strictly positive real number $\eta>0$, such that

$$
A^{T}(t) S+S A-2 C^{T} C \leq-\eta I ; \quad \forall t \geq 0
$$

where $A$ is given by Equation (5) and $I$ is the $(n \times n)$ identity matrix. The elements of $S$, i.e. $s_{i i}, s_{j}, i=1, \ldots, n$; $j=1, \ldots, n-1$, are given in function of $\alpha$ and $\beta$ defined in Equation (4).

\section{OBSERVER SYNTHESIS}

Consider the following dynamical system:

$$
\dot{\hat{x}}=f\left(u(t), u_{\tau}(t), \hat{x}(t), \hat{x}_{\tau}(t)\right)-\Lambda_{\rho} S^{-1} C^{T}(C \hat{x}(t)-y(t))
$$

where $S$ is given by Equation (6) and satisfies the precedent Lemma; $C$ is given by Equation (3);

$$
\hat{x}(t)=\left[\begin{array}{c}
\hat{x}_{1} \\
\hat{x}_{2} \\
\vdots \\
\hat{x}_{n}
\end{array}\right]
$$

and

$$
\hat{x}_{\tau}=\hat{x}(t-\tau)=\left[\begin{array}{c}
\hat{x}_{1 \tau} \\
\hat{x}_{2 \tau} \\
\vdots \\
\hat{x}_{n \tau}
\end{array}\right]
$$

$\hat{x}(t)$ and $\hat{x}_{\tau}(t)$ are the estimated state vectors, without and with delay respectively; $u$ and $y$ are the input and the output of the nonlinear system (1) respectively. The matrix $\Lambda_{\rho}$ is given in Equation (7).

One now states the following:

Theorem 1. Suppose that system (1) satisfies Assumptions (A1)-(A3). Then, for $\rho>\rho^{*}$ with $\rho^{*}=$ $\frac{4-3 \mu}{\eta(1-\mu)} c \lambda_{\max }(S)$, system (9) is an asymptotic observer for system (1). The observation error $\tilde{x}=\hat{x}(t)-x(t)$ is asymptotically stable for $\hat{x}(0), x(0) \in \mathbb{R}^{n}$.

Proof. Consider the observation error $\tilde{x}(t)=\hat{x}(t)-x(t)=$ $\left(\begin{array}{c}\tilde{x}_{1} \\ \tilde{x}_{2} \\ \vdots \\ \tilde{x}_{n}\end{array}\right)$ with $\tilde{x}_{i}=\hat{x}_{i}(t)-x_{i}(t) ; i=1, \ldots, n$.

Let $x^{i}=\left(\begin{array}{c}x_{1} \\ x_{2} \\ \vdots \\ x_{i}\end{array}\right), x_{\tau}^{i}=\left(\begin{array}{c}x_{1 \tau} \\ x_{2 \tau} \\ \vdots \\ x_{i \tau}\end{array}\right) ; \quad i=1, \ldots, n-1$, and

$\triangle f=\left(\begin{array}{c}\triangle f_{1} \\ \triangle f_{2} \\ \vdots \\ \triangle f_{n}\end{array}\right)$ where 


$$
\triangle f_{i}=\left\{\begin{array}{l}
f_{i}\left(u, u_{\tau}, \hat{x}^{i}, \hat{x}_{\tau}^{i}, x_{i+1}\right)-f_{i}\left(u, u_{\tau}, x^{i}, x_{\tau}^{i}, x_{i+1}\right) \\
; \quad i=1, \ldots, n-1 \\
f_{n}\left(u, u_{\tau}, \hat{x}, \hat{x}_{\tau}\right)-f_{n}\left(u, u_{\tau}, x, x_{\tau}\right) ; \quad i=n
\end{array}\right.
$$

For $i=1, \ldots, n-1$ we have:

$$
\begin{aligned}
f_{i}\left(u, u_{\tau}, \hat{x}, \hat{x}_{\tau}\right)-f_{i}\left(u, u_{\tau}, x, x_{\tau}\right) & =f_{i}\left(u, u_{\tau}, \hat{x}^{i}, \hat{x}_{\tau}^{i}, \hat{x}_{i+1}\right) \\
& -f_{i}\left(u, u_{\tau}, \hat{x}^{i}, \hat{x}_{\tau}^{i}, x_{i+1}\right) \\
& +\triangle f_{i}
\end{aligned}
$$

By applying the Mean Value Theorem:

$$
\begin{aligned}
f_{i}\left(u, u_{\tau}, \hat{x}, \hat{x}_{\tau}\right)-f_{i}\left(u, u_{\tau}, x, x_{\tau}\right) & =a_{i} \tilde{x}_{i+1}(t)+\triangle f_{i} \\
& ; i=1, \ldots, n-1
\end{aligned}
$$

where the functions $a_{i}=\frac{\partial f_{i}}{\partial x_{i+1}}\left(u, u_{\tau}, \hat{x}^{i}, \hat{x}_{\tau}^{i}, \lambda \tilde{x}_{i+1}+x_{i+1}\right)$; $i=1, \ldots, n-1$ verify (A2) with $\lambda \in[0,1]$ and $\triangle f_{i} ; i=$ $1, \ldots, n-1$ are given in (10).

The dynamics of the observation error $\tilde{x}$ is deduced from Equations (2) and (9):

$$
\dot{\tilde{x}}=f\left(u, u_{\tau}, \hat{x}, \hat{x}_{\tau}\right)-f\left(u, u_{\tau}, x, x_{\tau}\right)-\Lambda_{\rho} S^{-1} C^{T} C \tilde{x}
$$

By combining Equations (10),(11), (12) and (13), the dynamics of the observation error is:

$$
\begin{aligned}
\dot{\tilde{x}}(t) & =\left(A-\Lambda_{\rho} S^{-1} C^{T} C\right) \tilde{x}(t) \\
& +\triangle f\left(u(t), u_{\tau}(t), \hat{x}(t), x(t), \hat{x}_{\tau}(t), x_{\tau}(t)\right)
\end{aligned}
$$

where $A$ is an $(n \times n)$ matrix having the form given in Equation (5).

Let $\bar{x}=\Lambda_{\rho}^{-1} \tilde{x}$. It can be easily verified that $\Lambda_{\rho}^{-1} A \Lambda_{\rho}=\rho A$ and consequently

$$
\dot{\bar{x}}(t)=\rho\left(A-S^{-1} C^{T} C\right) \bar{x}(t)+\Lambda_{\rho}^{-1} \Delta f\left(u, u_{\tau}, \hat{x}, x, \hat{x}_{\tau}, x_{\tau}\right)
$$

Consider now the following Lyapunov-Krasovskii functional:

$$
V(\bar{x})=\bar{x}^{T} S \bar{x}+\frac{c \lambda_{\max }(S)}{1-\mu} \int_{t-\tau(t)}^{t}\|\bar{x}(s)\|^{2} d s
$$

where $c$ is the Lipschitz constant introduced in Assumption (A1), $\lambda_{\max }(S)$ is the largest eigenvalue of $S$ and $\mu$ is a constant introduced in Assumption (A3).

The time derivative of the functional $V$ is:

$$
\begin{aligned}
\dot{V} & =2 \rho \bar{x}^{T}\left(S A-C^{T} C\right) \bar{x}(t) \\
& +\frac{c \lambda_{\max }(S)}{1-\mu}\|\bar{x}(t)\|^{2}-\frac{c \lambda_{\max }(S)}{1-\mu}(1-\dot{\tau}(t))\|\bar{x}(t-\tau)\|^{2} \\
& +2 \bar{x}(t)^{T} S \Lambda_{\rho}^{-1} \triangle f\left(u, \hat{x}(t), x(t), \hat{x}_{\tau}(t), x_{\tau}(t)\right) \\
& =\rho \bar{x}(t)^{T}\left(S A+A^{T} S-2 C^{T} C\right) \bar{x}(t) \\
& +\frac{c \lambda_{\max }(S)}{1-\mu}\|\bar{x}(t)\|^{2}-\frac{c \lambda_{\max }(S)}{1-\mu}(1-\dot{\tau}(t))\|\bar{x}(t-\tau)\|^{2} \\
& +2 \bar{x}(t)^{T} S \Lambda_{\rho}^{-1} \triangle f\left(u, \hat{x}(t), x(t), \hat{x}_{\tau}(t), x_{\tau}(t)\right)
\end{aligned}
$$

By taking into account the precedent Lemma, the following inequality arises:

$$
\begin{aligned}
\dot{V} & \leq-\rho \eta\|\bar{x}(t)\|^{2} \\
& +\frac{c \lambda_{\max }(S)}{1-\mu}\|\bar{x}(t)\|^{2}-\frac{c \lambda_{\max }(S)}{1-\mu}(1-\dot{\tau}(t))\|\bar{x}(t-\tau)\|^{2} \\
& +2\left\|\bar{x}(t)^{T} S \mid\right\| \Lambda_{\rho}^{-1} \triangle f\left(u, \hat{x}(t), x(t), \hat{x}_{\tau}(t), x_{\tau}(t)\right) \|
\end{aligned}
$$

By using the Lipschitz condition described in Assumption (A1):

$\left\|\Lambda_{\rho}^{-1} \triangle f\left(u, \hat{x}(t), x(t), \hat{x}_{\tau}(t), x_{\tau}(t)\right)\right\| \leq c|| \bar{x}(t)\|+c\| \bar{x}(t-\tau) \|$ it follows that

$$
\begin{aligned}
\dot{V} & \leq-\rho \eta\|\bar{x}(t)\|^{2} \\
& +\frac{c \lambda_{\max }(S)}{1-\mu}\|\bar{x}(t)\|^{2}-\frac{c \lambda_{\max }(S)}{1-\mu}(1-\dot{\tau}(t))\|\bar{x}(t-\tau)\|^{2} \\
& +2 c\left\|S \left|\left\|\left|\bar{x}(t)\left\|^{2}+2 c|| S||\right\| \bar{x}(t)\|\| \bar{x}(t-\tau)\right)\right\|\right.\right.
\end{aligned}
$$

Since $\|\bar{x}(t)\| \| \mid \bar{x}(t-\tau))\left\|\leq \frac{1}{2}\left(\|\bar{x}(t)\|^{2}+\| \bar{x}(t-\tau)\right)\right\|^{2}$, then

$$
\begin{aligned}
\dot{V} & \leq-\rho \eta\|\bar{x}(t)\|^{2}+\frac{c \lambda_{\max }(S)}{1-\mu}\|\bar{x}(t)\|^{2} \\
& +3 c\left\|S \left|\left\|\left|\overline { x } ( t ) \left\|^{2}+\frac{\dot{\tau}(t)-\mu}{1-\mu} c|| S\left|\||| \bar{x}(t-\tau)\|^{2}\right.\right.\right.\right.\right.\right.
\end{aligned}
$$

It results from Assumption (A3) that $\frac{\dot{\tau}(t)-\mu}{1-\mu} \leq 0$, then

$$
\dot{V} \leq\left(-\rho \eta+\frac{4-3 \mu}{1-\mu} c \lambda_{\max }(S)\right)\|\bar{x}(t)\|^{2}
$$

Let $\rho^{*}=\frac{4-3 \mu}{\eta(1-\mu)} c \lambda_{\max }(S)$. If $\rho>\rho^{*}$ then:

$$
\dot{V} \leq-\alpha(\rho)\|\bar{x}(t)\|^{2}
$$

with $\alpha(\rho)=\rho \eta-\frac{4-3 \mu}{1-\mu} c \lambda_{\max }(S)$ and $\alpha(\rho)>0$, which results in $\dot{V} \leq 0$, that means $V(t) \leq V(0)$. Because of $\alpha(\rho)\|\bar{x}(t)\|^{2} \leq-\dot{V}$, it follows that:

$$
\alpha(\rho) \lim _{t \rightarrow+\infty} \int_{0}^{t}\|\bar{x}(s)\|^{2} d s \leq V(0)-\lim _{t \rightarrow+\infty} V(t)
$$

It results that $\lim _{t \rightarrow+\infty} \bar{x}(t) \rightarrow 0$, moreover $\|\tilde{x}(t)\| \leq \rho^{n}\|\bar{x}\|$ and in consequence $\lim _{t \rightarrow+\infty} \tilde{x}(t) \rightarrow 0$.

The observation error $\tilde{x}=\hat{x}(t)-x(t)$ is asymptotically stable for $\hat{x}(0), x(0) \in \mathbb{R}^{n}$ and then, system (9) is an asymptotic observer for system (1). This completes the proof. $\square$

Remark: If the time-delay in system (1) is constant, it corresponds to say that $\mu=0$ and from Theorem 1 , it can be easily deduced the following theorem:

Theorem 2. Suppose that system (1) satisfies Assumptions (A1)-(A3), then for $\rho>\rho^{*}$ with $\rho^{*}=\frac{4}{\eta} c \lambda_{\max }(S)$, system (9) is an asymptotic convergent observer for system (1). The observation error $\tilde{x}=\hat{x}(t)-x(t)$ is asymptotically stable for $\hat{x}(0), x(0) \in \mathbb{R}^{n}$.

\section{EXTENSION FOR SYSTEMS WITH MULTIPLE TIME-DELAY}

The observer design for a systems with multiple timedelays is the same as the above presented strategy. The 
only condition to preserve the same design is maintaining the triangular structure of the system nonlinearity with respect to each state of different time-delay. Consider the following triangular system with multiple time-delays:

$$
\left\{\begin{array}{l}
\dot{x}_{1}=f_{1}\left(x_{1}, x^{1 \tau}, x_{2}, u, u^{\tau}\right) \\
\dot{x}_{2}=f_{2}\left(x_{1}, x^{1 \tau}, x_{2}, x^{2 \tau}, x_{3}, u, u^{\tau}\right) \\
\vdots \\
\dot{x}_{i}=f_{i}\left(x_{1}, x^{1 \tau}, x_{2}, x^{2 \tau}, \ldots, x_{i}, x^{i \tau}, x_{i+1}, u, u^{\tau}\right) \\
\vdots \\
\dot{x}_{n-1}=f_{n-1}\left(x_{1}, x^{1 \tau}, \ldots, x_{n-1}, x^{(n-1) \tau}, x_{n}, u, u^{\tau}\right) \\
\dot{x}_{n}=f_{n}\left(x, x^{\tau}, u, u^{\tau}\right) \\
y=x_{1}
\end{array}\right.
$$

where

$$
\begin{gathered}
x^{i \tau}=\left(\begin{array}{c}
x_{i}\left(t-\tau_{1}\right) \\
x_{i}\left(t-\tau_{2}\right) \\
\vdots \\
x_{i}\left(t-\tau_{l}\right)
\end{array}\right) ; \quad i=1, \ldots, n \\
x^{\tau}(t)=\left(\begin{array}{c}
x^{1 \tau} \\
x^{2 \tau} \\
\vdots \\
x^{n \tau}
\end{array}\right)
\end{gathered}
$$

and

$$
u^{\tau}(t)=\left(\begin{array}{c}
u\left(t-\tau_{1}\right) \\
u\left(t-\tau_{2}\right) \\
\vdots \\
u\left(t-\tau_{l}\right)
\end{array}\right)
$$

where $\tau_{1}(t), \tau_{2}(t), \ldots, \tau_{l}(t)$ are $l$ known and bounded variable time-delays, each one satisfies the following assumption:

Assumption 4 (A4). The variable time-delay $\tau_{i}(t)$; $i=1, \ldots, l$ is bounded, that means $0<\tau_{i}(t) \leq \tau_{\max }^{i}$; $i=1, \ldots, l$, it is derivable and satisfies $\dot{\tau}_{i}(t) \leq \mu_{i}<1$; $i=1, \ldots, l$.

System (18) can be written in the following form:

$$
\left\{\begin{array}{l}
\dot{x}(t)=f\left(u(t), u^{\tau}(t), x(t), x^{\tau}(t)\right) \\
y(t)=C x(t)
\end{array}\right.
$$

where

$$
f=\left[\begin{array}{c}
f_{1}\left(x_{1}, x^{1 \tau}, x_{2}, u, u^{\tau}\right) \\
f_{2}\left(x_{1}, x^{1 \tau}, x_{2}, x^{2 \tau}, x_{3}, u, u^{\tau}\right) \\
\vdots \\
f_{n-1}\left(x_{1}, x^{1 \tau}, \ldots, x_{n-1}, x^{(n-1) \tau}, x_{n}, u, u^{\tau}\right) \\
f_{n}\left(x, x^{\tau}, u, u^{\tau}\right)
\end{array}\right]
$$

and the matrix $C$ is given in Equation (3).

Consider the following dynamical system:

$$
\dot{\hat{x}}=f\left(u(t), u^{\tau}(t), \hat{x}(t), \hat{x}^{\tau}(t)\right)-\Lambda_{\rho} S^{-1} C^{T}(C \hat{x}(t)-y(t))
$$

where $S$ is a matrix having the form given in Equation (6) and satisfies the precedent Lemma, $C$ is the matrix given in Equation $(3), \hat{x}(t)=\left[\begin{array}{c}\hat{x}_{1}(t) \\ \hat{x}_{2}(t) \\ \vdots \\ \hat{x}_{n}(t)\end{array}\right]$ and $\hat{x}^{\tau} \hat{x}(t-\tau)=\left[\begin{array}{c}\hat{x}^{1 \tau} \\ \hat{x}^{2 \tau} \\ \vdots \\ \hat{x}^{n \tau}\end{array}\right]$, are the estimated state vectors, without and with timedelay respectively, $u$ and $y$ are the input and the output of system (18) respectively, and finally, the matrix $\Lambda_{\rho}$ is given in Equation (7) with $\rho>0$.

The following theorem is an extension of Theorem 1, for the case of multiple time-delays.

Theorem 3. Suppose that system (18) satisfies Assumptions (A1)-(A4), then for $\rho>\rho^{*}$

with $\rho^{*}=\left(2+l+\sum_{i=1}^{l} \frac{1}{1-\mu_{i}}\right) \frac{c \lambda_{\max }(S)}{\eta}$, system $(20)$ is an asymptotic observer for system (18). The observation error $\tilde{x}=\hat{x}(t)-x(t)$ is asymptotically stable for $\hat{x}(0), x(0) \in \mathbb{R}^{n}$. The proof is deduced by following the same steps of the proof of the Theorem 1 . Define $\tilde{x}=\hat{x}-x$ and $\bar{x}=\Lambda_{\rho}^{-1} \tilde{x}$. The dynamics of $\bar{x}(t)$ is:

$$
\dot{\bar{x}}(t)=\rho\left(A-S^{-1} C^{T} C\right) \bar{x}(t)+\Lambda_{\rho}^{-1} \Delta f\left(u, u_{\tau}, \hat{x}, x, \hat{x}_{\tau}, x_{\tau}\right)
$$

Consider the following Lyapunov-Krasovskii functional:

$$
V(\bar{x})=\bar{x}^{T} S \bar{x}+c \lambda_{\max }(S) \sum_{i=1}^{l} \frac{1}{1-\mu_{i}} \int_{t-\tau_{i}(t)}^{t}\|\bar{x}(s)\|^{2} d s
$$

where $c$ is the Lipschitz constant introduced in Assumption (A1), $\lambda_{\max }(S)$ is the largest eigenvalue of $S$ and $\mu_{i}$; $i=1, \ldots, l$ is a constant introduced in Assumption (A4). The time derivative of $V$ is:

$$
\begin{aligned}
\dot{V} & =2 \rho \bar{x}^{T}\left(S A-C^{T} C\right) \bar{x}(t)+c \lambda_{\max }(S)\|\bar{x}(t)\|^{2} \sum_{i=1}^{l} \frac{1}{1-\mu_{i}} \\
& -c \lambda_{\max }(S) \sum_{i=1}^{l} \frac{1-\dot{\tau}_{i}(t)}{1-\mu_{i}}\left\|\bar{x}\left(t-\tau_{i}\right)\right\|^{2} \\
& +2 \bar{x}(t)^{T} S \Lambda_{\rho}^{-1} \delta f(u, \hat{x}(t), x(t)) \\
& =\rho \bar{x}(t)^{T}\left(S A+A^{T} S-2 C^{T} C\right) \bar{x}(t) \\
& +c \lambda_{\max }(S)\|\bar{x}(t)\|^{2} \sum_{i=1}^{l} \frac{1}{1-\mu_{i}} \\
& -c \lambda_{\max }(S) \sum_{i=1}^{l} \frac{1-\dot{\tau}_{i}(t)}{1-\mu_{i}}\left\|\bar{x}\left(t-\tau_{i}\right)\right\|^{2} \\
& +2 \bar{x}(t)^{T} S \Lambda_{\rho}^{-1} \delta f(u, \hat{x}(t), x(t))
\end{aligned}
$$

Considering the precedent Lemma, it follows that

$$
\begin{aligned}
\dot{V} & \leq-\rho \eta\|\bar{x}(t)\|^{2}+c \lambda_{\max }(S)\|\bar{x}(t)\|^{2} \sum_{i=1}^{l} \frac{1}{1-\mu_{i}} \\
& -c \lambda_{\max }(S) \sum_{i=1}^{l} \frac{1-\dot{\tau}_{i}(t)}{1-\mu_{i}}\left\|\bar{x}\left(t-\tau_{i}\right)\right\|^{2} \\
& +2\left\|\bar{x}(t)^{T} S \mid\right\|\left\|\Lambda_{\rho}^{-1} \delta f(u, \hat{x}(t), x(t))\right\|
\end{aligned}
$$

By using the Lipschitz condition described in Assumption (A1):

$$
\left\|\Lambda_{\rho}^{-1} \delta f(u, \hat{x}(t), x(t))\right\| \leq c\|\bar{x}(t)\|+c \sum_{i=1}^{l}\left\|\bar{x}\left(t-\tau_{i}\right)\right\|
$$

It results 


$$
\begin{aligned}
\dot{V} & \leq-\rho \eta\|\bar{x}(t)\|^{2} \\
& +c \lambda_{\max }(S)\|\bar{x}(t)\|^{2} \sum_{i=1}^{l} \frac{1}{1-\mu_{i}} \\
& -c \lambda_{\max }(S) \sum_{i=1}^{l} \frac{1-\dot{\tau}_{i}(t)}{1-\mu_{i}}\left\|\bar{x}\left(t-\tau_{i}\right)\right\|^{2} \\
& +2 c\left\|S \left|\|\bar{x}(t)\|^{2}+2 c\|S \mid\| \bar{x}(t)\left\|\sum_{i=1}^{l}\right\| \bar{x}\left(t-\tau_{i}\right) \|\right.\right.
\end{aligned}
$$

Since

$$
\left.\|\bar{x}(t)\| \sum_{i=1}^{l}\left\|\bar{x}\left(t-\tau_{i}\right)\right\| \leq \frac{1}{2}\left(l\|\bar{x}(t)\|^{2}+\sum_{i=1}^{l} \| \bar{x}\left(t-\tau_{i}\right)\right) \|^{2}\right)
$$

hence

$$
\begin{aligned}
\dot{V} & \leq-\rho \eta\|\bar{x}(t)\|^{2} \\
& +c \lambda_{\max }(S)\|\bar{x}(t)\|^{2} \sum_{i=1}^{l} \frac{1}{1-\mu_{i}} \\
& -c \lambda_{\max }(S) \sum_{i=1}^{l} \frac{1-\dot{\tau}_{i}(t)}{1-\mu_{i}}\left\|\bar{x}\left(t-\tau_{i}\right)\right\|^{2} \\
& +(2+l) c|| S \mid\|\bar{x}(t)\|^{2}+c\|S\| \sum_{i=1}^{l}\left\|\bar{x}\left(t-\tau_{i}\right)\right\|^{2}
\end{aligned}
$$

It follows that

$$
\begin{aligned}
\dot{V} & \leq-\rho \eta\|\bar{x}(t)\|^{2}+c \lambda_{\max }(S)\|\bar{x}(t)\|^{2}\left(2+l+\sum_{i=1}^{l} \frac{1}{1-\mu_{i}}\right) \\
& +c \lambda_{\max }(S) \sum_{i=1}^{l} \frac{\dot{\tau}_{i}(t)-\mu_{i}}{1-\mu_{i}}\left\|\bar{x}\left(t-\tau_{i}\right)\right\|^{2}
\end{aligned}
$$

From Assumption (A4): $\frac{\dot{\tau}_{i}(t)-\mu_{i}}{1-\mu_{i}} \leq 0$, then

$$
\dot{V} \leq\left(-\rho \eta+\left(2+l+\sum_{i=1}^{l} \frac{1}{1-\mu_{i}}\right) c \lambda_{\max }(S)\right)\|\bar{x}(t)\|^{2}
$$

Let $\rho^{*}=\left(2+l+\sum_{i=1}^{l} \frac{1}{1-\mu_{i}}\right) \frac{c \lambda_{\max }(S)}{\eta}$.

For $\rho>\rho^{*}$ it results:

$\dot{V} \leq-\xi(\rho)\|\bar{x}(t)\|^{2}$
where $\xi(\rho)=\rho \eta-\left(2+l+\sum_{i=1}^{l} \frac{1}{1-\mu_{i}}\right) c \lambda_{\max }(S)$ and $\xi(\rho)>0$, which results in $\dot{V} \leq 0$, that means $V(t) \leq V(0)$. Because of $\xi(\rho)\|\bar{x}(t)\|^{2} \leq-\dot{V}$, it follows that:

$$
\xi(\rho) \lim _{t \rightarrow+\infty} \int_{0}^{t}\|\bar{x}(s)\|^{2} d s \leq V(0)-\lim _{t \rightarrow+\infty} V(t)
$$

It results that $\lim _{t \rightarrow+\infty} \bar{x}(t) \rightarrow 0$, moreover $\|x(t)\| \leq \rho^{n}\|\bar{x}\|$ and in consequence $\lim _{t \rightarrow+\infty} x(t) \rightarrow 0$.
The observation error $\tilde{x}=\hat{x}(t)-x(t)$ is asymptotically stable for $\hat{x}(0), x(0) \in \mathbb{R}^{n}$. Hence, system (20) is an asymptotic observer for system (18). This complete the proof. $\square$

\section{SIMULATION EXAMPLE}

Consider the following nonlinear system:

$$
\left\{\begin{array}{l}
\dot{x}_{1}=2 x_{2}(t)+\cos \left(x_{2}(t)\right)-\frac{x_{1}(t-\tau)}{1+x_{1}(t-\tau)} \\
\dot{x}_{2}=-\frac{x_{2}(t-\tau)}{1+x_{2}(t-\tau)}+u(t-\tau) \\
y=x_{1}
\end{array}\right.
$$

The time-delay $\tau$ is considered constant. It can be seen that system (25) can be written in the form of system (1) with $f_{1}=2 x_{2}(t)+\cos \left(x_{2}(t)\right)-\frac{x_{1}(t-\tau)}{1+x_{1}(t-\tau)}$,

$f_{2}=-\frac{x_{2}(t-\tau)}{1+x_{2}(t-\tau)}+u(t-\tau)$ and $C=\left[\begin{array}{ll}1 & 0\end{array}\right]$.

Before applying Theorem 1, Assumptions (A1)-(A3) should be verified for system (25). It is easy to check that functions $f_{i}, i=1,2$ are Lipschitz, then Assumption (A1) is verified. It remains to verify Assumption (A2). According to Equation (4):

$$
a_{1}=\frac{\partial f_{1}}{\partial x_{2}}=2-\sin \left(x_{2}(t)\right)
$$

It results that $1 \leq a_{1} \leq 3$ then Assumption (A2) is satisfied with $\alpha=1$ and $\beta=3$. The elements of the matrix $S$ whose dimension is $2 \times 2$ are deduced from the precedent Lemma. These elements verify $\left|s_{1}\right|>\frac{\beta^{2} s_{11}^{2}}{2 \alpha}$ and $s_{22}>\frac{s_{1}^{2}}{s_{11}}$ (see Targui et al. (2002)). By taking $s_{11}=0,5$, the other elements can be deduced: $s_{1}=-2$ and $s_{22}=9$. The time-delay is considered constant at $\tau=1.30$. In this simulation, the input $u$ is given by the following equation

$$
u(t)=0,5+0,4 \sin (0,2 t+20) .
$$

The observer equations are:

$$
\left\{\begin{aligned}
\dot{\hat{x}}_{1} & =2 \hat{x}_{2}(t)+\cos \left(\hat{x}_{2}(t)\right)-\frac{\hat{x}_{1}(t-\tau)}{1+\hat{x}_{1}(t-\tau)} \\
& -18 \rho\left(\hat{x}_{1}-x_{1}\right) \\
\dot{\hat{x}}_{2} & =-\frac{\hat{x}_{2}(t-\tau)}{1+\hat{x}_{2}(t-\tau)}+u(t-\tau) \\
& -4 \rho^{2}\left(\hat{x}_{1}-x_{1}\right)
\end{aligned}\right.
$$

The initial conditions of the system (25) and the observer (27) are:

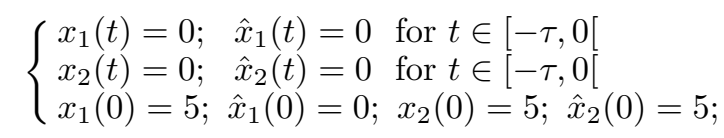

For this simulation, the tuning parameter $\rho$ is set to 10 . The value of this parameter allows to adjust the convergence time of the observer given in Equation (27).

Figures 1 and 2 show the states $x_{1}(t)$ and $x_{2}(t)$ and their estimated values $\hat{x}_{1}(t), \hat{x}_{2}(t)$; it can be seen that after a brief period of time $\hat{x}_{1}(t)$ converge to $x_{1}(t)$. On 


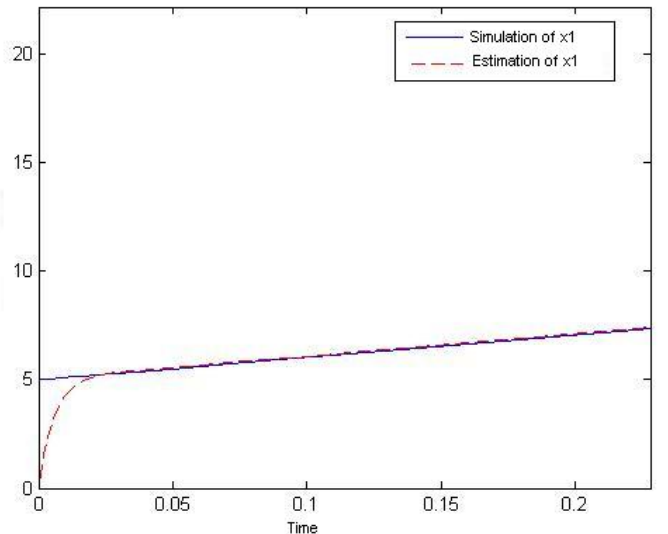

Fig. 1 . The state $x_{1}$ (solid line) and its estimated value $\hat{x}_{1}$ (dashed line).

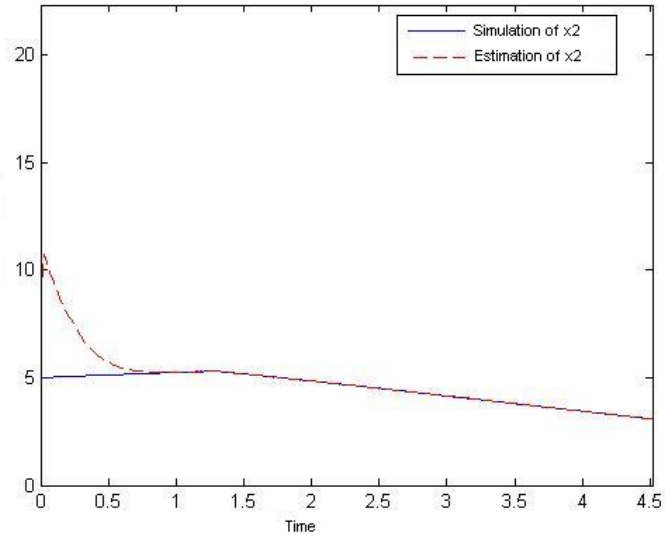

Fig. 2. The state $x_{2}$ (solid line) and its estimated value $\hat{x}_{2}$ (dashed line).

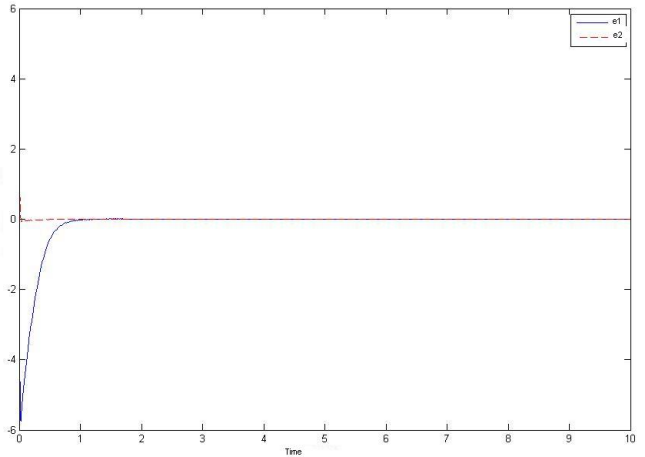

Fig. 3. The error estimation $e_{1}$ and $e_{2}$.

the other hand the time profile $x_{2}(t)$ and $\hat{x}_{2}(t)$ are quite similar. Figure 3 shows the errors estimations $e_{1}$ and $e_{2}$, after a small time $e_{1}$ and $e_{2}$ converge to zero. It can be appreciated that $\hat{x}_{2}(t)$ converges very quickly to $x_{2}(t)$.

\section{CONCLUSION}

In this work an observer for a class of delayed nonlinear triangular systems was presented. The time-delay is considered variable and it can be presented as a simple or multiple time-delay in the states or in the input of the system. The time-delay is supposed to be known and bounded. The main feature of the proposed observer with respect to others in the literature, is its simplicity. This is because (due to its particular structure) the system is treated in the original coordinates. Also, the observer gain is constant and does not require to solve any additional dynamical system. The observer is evaluated successfully through a simulation example.

\section{REFERENCES}

J.-P. Richard. Time-delay systems :an overview of some recent advances and open problems. Automatica, 39 :1667-1694, 2003.

M. Darouach. Linear Functional Observers for Systems with Delays in State Variables. IEEE Trans. Automatic Control, 46(3) :491-496, 2001.

A. Germani, C. Manes, et P. Pepe. A new approach to state observation of nonlinear systems with delayed output. IEEE Trans. Automatic Control, 47(1) :96-101, 2002

J.P. Gauthier, H. Hammouri, and S. Othman. A, A simple observer for nonlinear systems - application to bioreactors., IEEE Trans. on Aut. Control, 37:1992, pp 875-880.

J.P. Gauthier and I.A.K. Kupka, Observability and observers for nonlinear systems, SIAM J. Control. Optim., 32:1994 pp 994-994.

B. Targui, M. Farza, H. Hammouri,Observer design for a class of nonlinear systems, Applied Mathematics Letters, vol. 15, pp 709-720, 2002.

B. Targui, H. Hammouri, M. Farza, Observer design for a class of multi-output nonlinear systems : application to a distillation column, 40th CDC (IEEE Conference on Decision and Control),pp 3352-3357, 2001.

H. Hammouri, B. Targui and F. Armanet, High gain observer based on trianguler structure, Int. Journal on Robust and Nolinear Control., vol. 12, pp 497-518, 2002.

M. Farza, M. M'Saad and L. Rossignol, Observer desgin for a class of MIMO nonlinear systems, Automatica, vol. 40, pp 135-143, 2004.

S. Ibrir, Adaptive observers for time-delay nonlinear systems in triangular form Automatica, Volume 45, Issue 10, Pages 2392-2399, 2009.

M. Hou, P. Zitek, and R. J. Patton, An observer Design for Linear Time-dealy Systems, IEEE Transcations on Automatic Control, 47, 121-125, 2002.

Z. Wang, D. P. Goodall and K. J. Burnham, On designing observers for time-delay systems with nonlinear disturbances. International Journal of Control, 75(11), 803811, 2002. 